\title{
LA INVESTIGACIÓN APLICADA: UNA FORMA DE CONOCER LAS REALIDADES CON EVIDENCIA CIENTÍFICA
}

\author{
Zoila Rosa Vargas Cordero \\ Docente de la Maestría en Orientación de la \\ Universidad de Costa Rica \\ San José, Costa Rica
}

Recibido 1-VII-2008 • Aceptado 18-XI-2008 • Corregido 8-VI-2009

\begin{abstract}
Resumen: En este ensayo se expone una interpretación sobre la investigación aplicada vigente en el Sistema de Estudios de Posgrado de la Universidad de Costa Rica (SEP), para el trabajo final de graduación en las maestrías profesionales. Se discuten algunos criterios de la autora, discusiones académicas sobre el tema y otros razonamientos, con base en aportes de diferentes autores. Se fundamenta la necesidad de actualizar conocimientos acordes con la investigación y las posibilidades para realizarla, y se parte del supuesto de que la investigación es imprescindible en la formación profesional, ya que genera nuevos conocimientos que realimentan las disciplinas y el ejercicio profesional. La investigación requiere rigurosidad y excelencia; además, sostiene la plataforma para proponer y desarrollar nuevas investigaciones. Por ello, no se puede ignorar la importancia de la participación activa de estudiantes y docentes en los procesos de investigación, para favorecer la toma de conciencia de la realidad que se investiga, así como de los deberes y derechos de quienes investigan, lo que implica una nueva forma de pensar la práctica investigativa, de manera que se ofrezcan soluciones en función de los contextos, las acciones de las personas y los cambios filosóficos, ontológicos, epistemológicos y metodológicos en investigación. Al respecto, la investigación basada en el conocimiento y la comprensión de cómo perciben las personas su realidad y a si mismas; es decir, aquella que se enfoca en el desarrollo de acciones para el mejoramiento de la realidad que se investiga es vista a lo largo del ensayo como una necesidad operativa.
\end{abstract}

Palabras clave: Investigación, realidad compleja, investigación aplicada, evidencia científica.

\section{Introducción}

El Sistema de Estudios de Posgrado (SEP) de la Universidad de Costa Rica, estableció para las maestrías profesionales la investigación práctica aplicada para el proceso de graduación y estipula en el documento "Lineamientos de trabajos finales de investigación aplicada", que son las comisiones de cada Programa de posgrado las que establecen las características concretas de investigación que el estudiantado debe cumplir (Universidad de Costa Rica, SEP, 2007). La Maestría en Orientación ofrece el posgrado en el área, con mención profesional y atiende esos lineamientos; no obstante, lo que ellos proponen sobre la investigación práctica aplicada es limitado, especialmente para las ciencias sociales, por lo que es necesario unificar y consensuar los criterios a seguir al respecto.

La situación descrita conlleva la tarea de organizar ideas, conceptos y algunas referencias, con el fin de ofrecer a docentes y estudiantes un documento accesible, claro y sencillo, con una reorganización de elementos conceptuales que sirvan de 


\begin{abstract}
This is an essay where an interpretation of applied research that is current in the System of Studies of Post grade of the University of Costa Rica (SEP) is exposed, for the final work of graduation in the professional masters' degrees. Some criteria of the author, academic discussions are discussed on the subject and other reasonings, based in contributions of different authors. The need is to update knowledge in agreement with the investigation and the possibilities to carry it out, and is based on the assumption that research is essential in the professional formation, since it generates new knowledge that feedback the disciplines and the professional exercise. The research itself, on one hand requires rigor and excellence; and in the other, it maintains the platform to propose and to develop new investigations. For that reason, the importance of active participation of students and teachers in the investigation processes cannot be ignored, to favor the taking of conscience of the reality that is investigated, as well as of the duties and rights of those who they investigate, which implies a new form to think the practice of research, so that solutions based on the context of people's actions and on philosophical, ontological, epistemological and methodological changes in investigation are offered. On that matter, research based on the knowledge and the understanding of how people perceive their reality and themselves; that is to say, that which focuses on the development of actions for the improvement of the reality that is investigated is analyzed throughout the essay as an operative necessity.
\end{abstract}

Key words: Research, complex reality, applied research, science. base para fundamentar los principios de la investigación práctica, empírica o aplicada -como también se la conoce-, que puedan ser empleados en la Maestría profesional en Orientación y en otras donde las respectivas Comisiones de Programas de Posgrado lo consideren pertinente.

Para comprender la relevancia de la investigación aplicada en el campo de la Orientación, se parte de la forma cómo se entiende la realidad en la disciplina; luego, se argumentan razones importantes de por qué y para qué investigar en Orientación. Asimismo, se hace referencia a lo compleja que resulta la investigación como tal, para enfocar el ensayo propiamente en lo que es investigación aplicada, con una breve mención de algunos tipos de investigación clasificados en la nomenclatura práctica, aplicada o empírica, como se le reconoce y que, por lo tanto, se pueden realizar en posgrado. Finalmente, se presentan las conclusiones sobre la investigación aplicada vista como un proceso investigativo científico, serio y riguroso, y como una forma necesaria y óptima para conocer las realidades desde la evidencia misma.

\section{Cómo entender las realidades}

En la disciplina de Orientación se busca hacer, junto con las personas, un trabajo integrador que atienda el ser humano en todas sus dimensiones, la diversidad y el contexto en que se desarrollan; sin embargo, aún en las mismas áreas de la disciplina existen tendencias hacia los saberes parcelados o compartimentados. En consecuencia, se diluyen los conjuntos complejos entre las partes y el todo (Morín, 2001). Por esa razón, se mantiene un enfoque reduccionista, al considerar solo ciertos factores para solucionar los problemas de manera integral, que dificulta ver la realidad desde un ámbito global y complejo, así como verlas, sobre todo, desde la evidencia directa.

La complejidad existe cuando no se separan los componentes que constituyen 
un todo o cuando se observa un todo como tal y los elementos que lo influyen. Para Cerda (1997), la totalidad es un todo concreto, que se puede definir como la síntesis de pluralidad y unidad. Hace referencia al significado que tiene en el trabajo metodológico y operativo de la investigación comunitaria donde, para su comprensión, esa totalidad tiene valor de contexto, de circunstancia o de hecho. Es decir, es la totalidad de "alguien", tomando en cuenta que toda unidad está llena de contradicciones, por lo que no existen los fenómenos sociales lineales, sino que hay que estudiarlos y comprenderlos en la realidad dada, con los aspectos dinámicos, las fuerzas y variables que lo influyen.

Un hecho que no puede escapar a la investigación en general, y en el campo de la Orientación en particular, es la globalización que caracteriza nuestro tiempo y conduce, con mayor frecuencia que en épocas anteriores, a la complejidad. Una forma de generar conocimiento y soluciones en realidades complejas, cuyos contextos son complejos a su vez, es la investigación aplicada. Por ello, la investigación en Orientación debe situar a la persona y el acontecimiento en su contexto, comprender cómo es modificado, tomar en cuenta todos los elementos que forman parte de él e identificar relaciones entre la situación puntual y el contexto, de manera que las soluciones a los acontecimientos-problema se produzcan, bajo el enfoque de pensamiento complejo (Dugarte, 2006).

La complejidad individual se encuentra presente en la complejidad social. Los seres humanos han evolucionado durante siglos y épocas en las que se han desarrollado diversas teorías, ideologías y filosofías; pero, paralelamente, éstas y la humanidad han cambiado, de ahí que sea necesario superar dogmas, estereotipos y estructuras, para entender la realidad sin pre-concepciones ni estructuras rígidas. La actitud científica puede hacer cambiar la visión de mundo que se posee, renovarla y actualizarla hasta llevar a descartar mitos, revisar tradiciones y mantener una perspectiva más realista de la vida humana.

Personas distintas en diversos contextos constituyen similitudes y diferencias, contradicciones e inconsistencias, porque se parecen tanto como se diferencian en pensamientos, sentimientos y formas de actuar. Esto se traduce hoy en la perspectiva de la realidad social como "realidad compleja". Por ello, no se puede hablar de causa y efecto, porque las cogniciones, percepciones, emociones, tradiciones, culturas, y formas de ver la vida no producen efectos, aprendizajes, implicaciones o consecuencias similares en cada persona.

En las ciencias sociales, en general, y en Orientación, en particular, se pueden cuantificar algunas variables susceptibles de ser medidas, se pueden utilizar métodos cuantitativos para, entre otras cosas, diagnosticar o generalizar resultados; no obstante, la visión lineal de la causalidad y universalidad de sus conclusiones resulta poco adecuada para el análisis de los problemas sociales. De igual forma, existen variables y condiciones que únicamente se pueden analizar en forma cualitativa, por las relaciones y diferencias existentes entre las personas, los contextos y los grupos sociales. Pese a ser diferentes, los enfoques cuantitativo y cualitativo de investigación no son excluyentes. Cívicos y Hernández (2007) mencionan que la investigación mixta combina las ventajas de ambos y emplea diferentes técnicas para la recogida de información, lo que permite aproximarse mejor a la complejidad propia de la realidad social.

Si se asume que la realidad social no es lineal ni homogénea, sino que está compuesta por elementos dinámicos y variables, desde el enfoque fenomenológico se considera que la realidad es compleja e incontrolable y su conocimiento no es universal, sino que se entiende e interviene desde un contexto específico. Así, por ejemplo, un problema de investigación se enmarca dentro de un contexto para 
ubicarlo como una realidad particular, un todo, tomando en cuenta la heterogeneidad y el cambio constante y dinámico de personas, fenómenos y circunstancias, o lo que es lo mismo, la unidad y pluralidad contempladas a la vez.

Situar a la persona en un contexto, visto como el medio al que pertenece, integrado por la cultura y en la cultura la humaniza, observa, percibe, aprende y actúa; por lo tanto, es el que crea una realidad propia que puede ser intervenida, mejorada o transformada por quien investiga, emplea la práctica como investigación y ésta, a su vez, como práctica. Para Cívicos y Hernández (2007), la investigación aplicada o práctica se caracteriza por la forma en que analiza la realidad social y aplica sus descubrimientos en la mejora de estrategias y actuaciones concretas, en el desarrollo y mejoramiento de éstas, lo que, además, permite desarrollar la creatividad e innovar.

\section{3. ¿Por qué y para qué investigar?}

Investigar es conocer, transformar la tradición y el rutinario quehacer hacia nuevas visiones de mundo contextualmente realistas. Una disciplina sin producción científica se debilita y al no haber avance científico pierde identidad y corre el riesgo de desaparecer como tal. Si eso ocurre, al mismo tiempo, se empobrecen las características del perfil profesional y su quehacer se vuelve rutinario. Estar atentos a lo que se observa, a lo que pasa en la realidad del campo disciplinar es una forma de participar y dialogar dentro de la disciplina y es una forma de propiciar inquietudes que se pueden refrendar y comparar, promoviendo la participación e influyendo o interviniendo en los procesos humanos y sociales. Investigar es buscar y producir conocimiento de manera organizada, imparcial y sistemática, de ahí su importancia.
La adopción universal de una actitud científica, para Bunge (1975), puede hacer personas más sabias, cautas en la recepción de información y en admitir creencias; más exigentes en la constatación de opiniones propias y tolerantes con las de otros. Estar en disposición de eliminar mitos consagrados daría una visión de mundo "eternamente joven", basada en teorías contrastadas, en vez de tradiciones que rehúyen tenazmente toda contrastación con los hechos. Ello sustentaría una visión realista de la vida y realidad humana.

La investigación permite cuestionar, reflexionar y actuar sobre el acontecer histórico y social en la medida que favorece un criterio propio, fundamentado científicamente; criterio que beneficia a poblaciones atendidas, la creación novedosa de estrategias y métodos de intervención, el aumento de la calidad de la investigación, el rendimiento y respeto de la imagen profesional. Un posicionamiento científico de las personas profesionales y en la disciplina misma se logra con productos o resultados rigurosos y sistematizados obtenidos de la investigación. Como ventaja adicional, el avance científico determina una mayor valoración externa de la profesión y el ejercicio profesional, especialmente cuando en los ambientes laborales - por la índole de su quehacer-, se dan debates, defensas o discusiones con base en criterios y evidencia científica.

Al hacer investigación, las personas conocen mejor la disciplina, porque deben describir, interpretar y analizar fenómenos sociales y humanos en contextos reales y, por ende, su práctica profesional se vuelve más eficiente y efectiva al formular y ejecutar intervenciones y proyectos novedosos y creativos, apropiados a circunstancias y necesidades concretas. Gracias a la investigación, toda persona investigadora mejora su práctica, innova y se desarrolla con seguridad y profesionalismo, favoreciendo a la comunidad e institución para la cual labora. La importancia del "por qué" y "para $q u e ́$ " de la investigación en Orientación, 
queda abierta a partir de ahora para que cada lector o lectora opine al respecto.

\section{La investigación}

Mucho se ha dicho sobre lo densa y compleja que es la investigación, sobre todo cuando se realiza por primera vez, porque se la considera una actividad para especialistas que poseen un conglomerado de conocimientos sobre paradigmas, posturas epistemológicas, metodológicas, técnicas e instrumentos de investigación. Además, mucho se ha escrito sobre diferentes tipos de investigación entre disciplinas, así como terminologías específicas. El abordaje de la gran cantidad de posturas en investigación hace que el estudiantado de maestrías profesionales generalmente se confunda más que se aclare; pese a que, obviamente, la investigación se pueda hacer de muchas maneras, diferenciadas por los marcos teórico-metodológicos que se utilicen en cada caso. Sin embargo, al margen de cuál se adopte, es la rigurosidad científica la que determina la validez de los resultados y la ampliación del conocimiento científico, con lo que se crean nuevas teorías o se modifican las ya existentes (Cerda 1997).

Algunos autores mencionan dos tendencias para investigar. La primera es la investigación básica -también conocida como investigación fundamental, exacta o investigación pura-, que se ocupa del objeto de estudio sin considerar una aplicación inmediata, pero teniendo en cuenta que, a partir de sus resultados y descubrimientos, pueden surgir nuevos productos y avances científicos (Cívicos y Hernández, 2007; Padrón, 2006). La segunda es la investigación aplicada, entendida como la utilización de los conocimientos en la práctica, para aplicarlos en provecho de los grupos que participan en esos procesos y en la sociedad en general, además del bagaje de nuevos conocimientos que enriquecen la disciplina. Al respecto, en las ciencias puras y la investigación básica se busca indagar cómo funcionan las cosas para un uso posterior, mientras en las ciencias prácticas la investigación aplicada tiene como propósito hacer un uso inmediato del conocimiento existente.

Como se puede observar, uno de los signos de la época actual es la multiplicidad de posturas científicas en cuanto a los saberes, filosofías, enfoques, disciplinas, especialidades, teorías, métodos y técnicas de investigación. Esto lleva la necesidad de repensar la coordinación en la formación profesional, para unificar criterios y establecer una profunda unión e integración con base en el diálogo, para poner en común "verdades" distintas sobre la realidad que se investiga, que permita, a su vez, descubrir significados a partir de la discusión dentro de comunidades científicas (Martínez, 2004).

\section{La investigación aplicada}

Para Murillo (2008), la investigación aplicada recibe el nombre de "investigación práctica o empírica", que se caracteriza porque busca la aplicación o utilización de los conocimientos adquiridos, a la vez que se adquieren otros, después de implementar y sistematizar la práctica basada en investigación. El uso del conocimiento y los resultados de investigación que da como resultado una forma rigurosa, organizada y sistemática de conocer la realidad.

Con el fin de ofrecer un referente comprensible de la expresión "investigación aplicada", se exponen algunas de las ideas de Padrón (2006) al respecto, para quien la expresión se propagó durante el siglo XX para hacer referencia, en general, a aquel tipo de estudios científicos orientados a resolver problemas de la vida cotidiana o a controlar situaciones prácticas, haciendo dos distinciones:

a. La que incluye cualquier esfuerzo sistemático y socializado por resolver problemas o intervenir situaciones. 
En ese sentido, se concibe como investigación aplicada tanto la innovación técnica, artesanal e industrial como la propiamente científica.

b. La que sólo considera los estudios que explotan teorías científicas previamente validadas, para la solución de problemas prácticos y el control de situaciones de la vida cotidiana.

La historia de la investigación científica muestra el aprovechamiento de productos teóricos para el diseño de sistemas de acción eficientes para resolver alguna necesidad o situación social deficiente, mejorable de algún modo. Para el caso de las Ciencias Sociales, se mencionan el entrenamiento mediante la teoría conductista, la psicoterapia basada en teorías lingüísticas, entre otros. En ese sentido, sólo es investigación aplicada aquella que se enmarca dentro de una secuencia programática de búsquedas que tienen como núcleo el diseño de teorías científicas. La idea de fondo está en las relaciones de utilidad del conocimiento, para resolver problemas e intervenir situaciones concretas, por lo que se considera que la función elemental del conocimiento en los organismos va estrechamente asociada a las necesidades de subsistencia, mediante mecanismos de adaptación y control del medio.

El concepto de investigación aplicada tiene firmes bases tanto de orden epistemológico como de orden histórico, al responder a los retos que demanda entender la compleja y cambiante realidad social. El fundamento epistemológico de esta expresión está en la base de distinciones tales como "saber y hacer", "conocimiento y práctica", "explicación y aplicación", "verdad y acción". Asimismo, exige una estructura metodológica y comunicacional-documental diferente a la de la investigación descriptiva y explicativa. Los análisis y normativas institucionales están en la obligación de hacer esas diferencias, evitando la imposición de los mismos esquemas metodológicos y documentales para todo tipo de investigación.
En el marco de la Sociología de la Ciencia, esa es una tendencia reciente que minimiza las fronteras entre lo teórico y lo aplicativo, que propugna una vinculación inseparable entre el "saber y el hacer", pretendiendo que toda investigación teórica se enfoque en sus aplicaciones. Un ejemplo significativo de esto es la "Investigaciónacción", donde la teoría se construye a partir de necesidades y búsquedas prácticas.

$\mathrm{Al}$ retomar las tendencias mencionadas de la investigación, se indican las particularidades metodológico-estructurales y documentales de la investigación aplica$\boldsymbol{d a}$, ya que en ellas se sigue una lógica diferente a la de enfoques de investigación de las otras fases de desarrollo de la ciencia, sin que por ello deje de cumplir con la necesaria rigurosidad teórico-metodológica, que subyace al quehacer científico.

Murillo (2008) afirma que un gran número de estudios participa de la naturaleza de la investigación básica y aplicada, puesto que toda investigación involucra problemas teóricos y prácticos. Por su parte, Denzin (citado por Cerda, 2007) propone utilizar la triangulación para combinar métodos y hacer posible un tipo de "investigación total", donde la persona que investiga "... examine un problema (o unos problemas) desde tantas perspectivas metodológicas, epistemológicas y técnicas como le resulte posible. Cada método implica una línea de acción diferente hacia la realidad" (p. 107).

En cuanto a sus instancias de desarrollo, la investigación aplicada sigue una estructura general. Debido a su misma naturaleza, el concepto de 'problema de investigación' es diferente. Mientras en los otros enfoques de investigaciones el problema es de orden cognitivo, en ellas es de orden práctico, ya que se trata de una situación dada o deficitaria que puede ser mejorada. No obstante, la investigación aplicada se encuentra estrechamente vinculada con la investigación básica, pues depende de los principios científicos de esta última para su ejecución. Esto queda aclarado cuando se comprende que la investigación aplicada, al 
igual que en otros enfoques, requiere de un marco teórico, que, en este caso, significa la selección de una teoría que se expone en sus conceptos centrales y sus rasgos contextuales acordes a la situación problema identificada.

Aunque en los lineamientos para los trabajos finales de graduación, el Sistema de Estudios de Posgrado de la Universidad de Costa Rica (p. 1) menciona que "La investigación práctica aplicada se da a través de: estudios de casos, diagnósticos y propuestas, producción artística o documental, laboratorios, prácticas profesionales, etc.”; para la Maestría de Orientación ha sido necesario legitimar una estructura que sirva de guía o referente a las poblaciones cursantes, en los procesos de investigación. Al respecto, se acordó asumir como guía o referente de la Maestría en Orientación, la estructura que propone Padrón (2006), para quien la investigación aplicada tiene como finalidad la búsqueda y consolidación del saber, la aplicación de los conocimientos para el enriquecimiento del acervo cultural, y científico, así como para la producción. Ésta comprende, en principio, los siguientes pasos:

1. Partir de una situación problemática que requiere ser intervenida y mejorada. Se debe describir sistemáticamente esa situación problema, de manera que se justifique con criterios relevantes su orden práctico.

2. Seleccionar una teoría, para luego exponerla en sus conceptos centrales y en sus rasgos contextuales.

3. Examinar la situación "problema" a la luz de la teoría seleccionada, de ésta se deriva un prototipo de acción, con el cual se busca resolver favorablemente la situación "problema”. Obviamente en él se contempla la descripción sistemática con sus secuencias e instrumentaciones pues resultará ser el método y/o un modelo a emplear y comprobar en este proceso práctico aplicado.
4. Ensayar y probar el prototipo descrito como paso 3, para determinar la probabilidad que tiene el modelo aplicativo para resolver la situación problema (Universidad de Costa Rica, Facultad de Educación, 2007).

Una vez cumplidos los pasos de la estructura, es imprescindible completar el proceso de análisis de la práctica investigativa, detallar los resultados obtenidos y, por último, elegir el formato adecuado para presentar el informe final escrito, sin obviar la defensa oral y pública tal como lo establece en este caso, el Reglamento del SEP. Es así como se muestra, no sólo la guía de estructura a seguir sino también, la importancia de este tipo de investigación, en la cual a la persona o institución que investiga, lo que le interesa son las consecuencias prácticas de los hallazgos de la investigación para su aplicación inmediata o futura, enmarcada en la evidencia de una realidad dada. Razones por las que recientemente algunos organismos que financian investigación están prefiriendo la investigación aplica$\boldsymbol{d} \boldsymbol{a}$ sobre la investigación básica, descriptiva y explicativa (Padrón 2006).

\section{Tipos de investigación práctica aplicada}

A continuación, se describen los tipos de investigación mencionados por el SEP relacionados con la Orientación, así como de otros que por sus propósitos y métodos cumplen con lo que se ha denominado como investigación aplicada o práctica.

Las Investigaciones prácticas enfocadas en diagnósticos implican un procedimiento llevado a cabo mediante encuestas, entrevistas o cuestionarios, para establecer las necesidades o problemas que afectan un sector o una situación de la realidad social y que es motivo de estudio o investigación. Responden con propuestas que tienen que ver con producción, tales como: definir políticas institucionales, 
lineamientos y reglamentos específicos; la producción de documentos de acceso y propuestas para el desarrollo de prácticas en instituciones u organizaciones; la producción de materiales y herramientas técnicas especializadas; $y$, documentar buenas prácticas de intervención, producir métodos y técnicas de evaluación.

Los estudios de casos, cuyo pionero fue Sigmund Freud, no están limitados a las personas, sino que son un método de investigación que se emplea como práctica regular para estudiar rigurosamente, y paso a paso, los diversos factores que producen desarrollo, cambio o afectan una situación dada de un problema social determinado. El objeto de estudio puede ser un negocio, una familia, escuela, pandilla, grupo, u organización social, entre otras. Salkind (1999) hace una diferencia entre los estudios de caso, en términos del estudio de grupos y de caso único, con la particularidad de que este último se enfoca en un solo individuo o cosa, y lo define como “... un método empleado para estudiar un individuo o una institución en un entorno o situación única y de una forma lo más intensa y detallada posible" (p. 211). No obstante, en ambos tipos de estudio de caso se revela una diversidad y riqueza de conductas, además de sugerir direcciones para otras acciones o estudios.

Las prácticas, entendidas como investigación aplicada, son experiencias de investigación con propósitos de resolver o mejorar una situación específica o particular, para comprobar un método o modelo mediante la aplicación innovadora y creativa de una propuesta de intervención, en este caso de índole Orientadora, en un grupo, persona, institución o empresa que lo requiera. Por su parte, Boggino y Rosekrans (2004) y Cívicos y Hernández (2007) mencionan la investigación-acción y la investigación participativa como aquellas que responden a aplicaciones, de ahí que sean vistas como prácticas de investigación diferentes de la investigación práctica aplicada.
La investigación-acción es un concepto acuñado por Lewin, quien buscaba algo útil, inmediato y aplicable para grupos sociales especialmente en desventaja. Para Corey (citado por Boggino y Rosekrans, 2004, p. 26), "la investigación en acción es un proceso a través del cual los prácticos intentan estudiar sus problemas científicamente a fin de guiar, corregir y evaluar sus decisiones y acciones", que refiere a la totalidad de acciones que desarrolla el investigador como sujeto del conocimiento, quien las traslada al grupo, como sujetos que poseen un mayor nivel de integración (Samaja, 2002).

La investigación-acción busca cambios en la práctica para mejorar en términos funcionales. Su interés es reflexionar sobre la práctica y la postura epistemológica y teórica que le sustenta. Se utilizan distintos recursos que permiten clarificar para definir el tema de interés y diagnosticar las debilidades más significativas, las cuales deben ser una situación o problema que sea factible de incidir en un cambio. La persona que investiga debe plantear un problema relacionado con su quehacer, cuyo objetivo sea mejorar la práctica educativa o pedagógica y es en el ámbito educativo donde más se emplea. Puede ser ejecutada de manera individual o en equipos de trabajo. Ha sido clasificada por Latorre (citado por Boggino y Rosekrans, 2004) bajo la modalidad hermenéutica como investigación práctica.

La investigación participativa se fundamenta en identificar necesidades propias de un colectivo, con fines claros de que se analicen críticamente, para que las personas entiendan sus causas y encuentren las acciones necesarias para superarlas. En la investigación participativa se combina y relaciona la investigación con la práctica, configurando un universo de trabajo con objetivos claramente definidos (Vargas, 1991). Es una estrategia de investigación de movimiento dinámico, que busca alternativas concretas acerca de cómo generar los procesos de reflexión 
que permitan corregir distorsiones en las propias creencias y errores en la forma de resolver los problemas en la práctica. Esto puede conducir a la persona interesada a una crítica y toma de conciencia de su papel en la sociedad y del tipo de acciones que desea realizar, o cambiar.

La investigación participativa, vista como acto de conocimiento desde una concepción práctica de la investigación, cuenta con los aportes de las personas investigadoras y de los grupos como sujetos cognoscentes, cuyo objeto por descubrir es la realidad concreta. El pionero de este enfoque fue Paulo Freire, quien afirmó que cuanto más se profundiza con los grupos investigados, tanto más se puede superar el conocimiento anterior (Cerda, 1991).

Por su poco uso y relevancia, es importante hacer especial referencia a lo que desde décadas anteriores se denominó el método de investigación protagónica, muy semejante a la investigación-acción y a las características de investigación aplicada. Toma parte en la investigación educativa y pretende que la persona realice una investigación sobre sí misma, su práctica y los principios teórico-prácticos que la rigen, por lo que se convierte en sujeto y objeto de estudio. Utilizando el sentido común, se llega a conocer y a reflexionar de manera rigurosa y sistemática sobre la propia práctica, con el fin de rectificar aspectos y elaborar opciones activas de cambio y superación.

La investigación evaluativa aplica conocimiento científico con información objetiva para acumular evidencia válida $\mathrm{y}$ confiable, a fin de precisar en qué medida es posible tomar decisiones para la transformación de programas sociales. Puede tener como finalidad cambiar actitudes, conocimientos y conductas de personas; o bien, de instituciones o comunidades. Refiere a un valor de utilidad y a una lógica científica -en cuyo caso se orienta a precisar el proceso de juzgar, al establecer criterios claros y precisos para lograr el valor de una verdad-. Permite mayor cualificación y el mejoramiento de los programas sociales (Weiss, 1987). La evaluación puede ser de tipo cuantitativa o cualitativa, pero siempre responde a una lógica de acción al contribuir a la toma de decisiones subsiguientes, así como a mejorar la marcha futura.

Cabe mencionar que la investigación aplicada refiere al empleo de otros tipos de estudio y técnicas, entre las que se mencionan: estudios de mercado, sondeos de opinión pública, entrevistas y grupos focales. Todos con miras a responder con propuestas estratégicas de mejoramiento o cambio de una situación problema o para documentar experiencias basadas en situaciones reales.

\section{Consideraciones finales}

La investigación aplicada constituye un enlace importante entre ciencia y sociedad. Con ella, los conocimientos son devueltos a las áreas de demanda, ubicadas en el contexto, donde se da la situación que será intervenida, mejorada o transformada. Al prescindir de este tipo de investigaciones se aísla el conocimiento científico de los contextos donde interviene, quedando cada profesional en manos del acontecer externo y de teorías ajenas a la evidencia de la realidad que enfrenta en su práctica profesional. La investigación como actividad científica resulta ser densa y compleja, propia de personas especializadas; no obstante, para personas nóveles en este quehacer o aquellas que cursan Maestrías Profesionales, por sus características, la investigación aplicada constituye una forma ágil y placentera de resolver situaciones problema de interés propio del ámbito disciplinar y profesional.

Si bien es difícil demostrar la prioridad o exclusividad de un método, tipo o modelo de investigación para la interpretación o comprensión de una realidad específica, resulta de fácil entendimiento que todo ser humano ha crecido en contextos, bajo situaciones socio-históricas diferentes, que 
implican creencias, intereses, necesidades, fines, temores y otros más, propios de las experiencias, formación y educación de cada uno. Cada ser humano vive y percibe desde el mismo contexto distintas coordenadas, dando como resultado "verdades" $y$ "realidades" diferentes y complejas, como claras razones que confirman que para obtener resultados desde la evidencia científica contextual resulta adecuada la investigación práctica aplicada.

Promover este tipo de investigación es una manera de impulsar la cultura de investigación en la formación de posgrado bajo la modalidad de maestría profesional, puesto que, al haber mayor divulgación de conocimientos basados en la evidencia de realidades dadas, se comprueba la utilidad inmediata de la aplicación, se incentiva la función investigativa, se eleva el estatus de la intervención y, para el caso que nos ocupa, el rango de la Orientación como disciplina.

Fomentar la publicación en revistas científicas se logra con la sistematización de las prácticas profesionales realizadas como investigación. De manera que estas experiencias se pueden aprovechar para dar un salto en la formación de posgrado, por medio del desarrollo de una cultura de divulgación de nuevos conocimientos, generados a partir de la reflexión crítica sobre la propia acción, vista como objeto de estudio durante el proceso de la práctica investigativa.

La investigación aplicada enmarcada en una agenda programática resulta mucho más susceptible de ser controlada y gestionada. Además de ser una forma de mantenerse en constante actualización sobre realidades contextuales, metodológicas y nuevas teorías. De ahí que con la investigación aplicada se benefician las prácticas profesionales y quien las ejecuta, al ofrecer sistemáticamente un rendimiento de cuentas, con lo que se favorece no sólo la población que se atiende, sino también la institución.

A modo de conclusión cabe mencionar la importancia de experimentar procesos de investigación aplicada y de asumir la práctica como objeto de estudio en sí misma, objeto de análisis, reflexión e intervención, con responsabilidad ética y profesional. Es importante desarrollar procesos de esta índole, porque favorecen el análisis riguroso de lo producido e implican la búsqueda bibliográfica para extraer de una teoría, los aspectos aplicables para la situación problema en una realidad contextual. Elegir el escenario que sirve de fuente de información y observación, es clave para la aplicación de modelos, estrategias e instrumentos por ser empleados en la práctica orientadora y en el área de interés, para mejorar la calidad de la atención que, como profesionales, brindan a las poblaciones en sus distintos entornos.

Trabajar con criterios claros sobre la propia acción, además de la fundamentación científica, elevan, sin lugar a dudas, el rango de la disciplina no sólo por la producción científica, sino porque esto impulsa la formación constante y permanente de hacer investigación en el gremio de profesionales de la Orientación.

\section{Referencias bibliográficas}

Boggino, N. y Rosekrans, K. (2004). Investigación-acción: Reflexión critica sobre la práctica educativa. Rosario: Homo Sapiens.

Bunge, M. (1975). La investigación científica. Barcelona: Ariel.

Cerda, H. (1991). Los elementos de la investigación: Cómo reconocerlos, diseñarlos y construirlos. Colombia: Editorial El Búho.

Cerda, H. (1997). La investigación total. La unidad metodológica en la investigación científica. Colombia: Editorial Magisterio.

Cívicos, A. y Hernández, M. (2007). Algunas reflexiones y aportaciones en torno a los enfoques teóricos y prácticos de 
la investigación en trabajo social. Revista Acciones e investigaciones sociales, 23, 25-55.

Dugarte, A. (2006). Repensar en la investigación educativa de la nueva era. (PonenciapresentadaenlaIIIJornada de Investigación Humanística y Educativa de la Facultad de Ciencias de la Educación). Revista Ciencias de la Educación, 1(27), 99-108.

Martínez, M. (2004). Los grupos focales de discusión como método de investigación. Heterotopía, 26, 59-72.

Morín, E. (2001). Los siete saberes necesarios para la educación del futuro. Buenos Aires, Argentina: Ediciones Nueva Visión.

Murillo, W. (2008). La investigación científica. Consultado el 18 de abril de $2008 \mathrm{de} \mathrm{http//www.monografias.com/}$ trabajos15/invest-científica/investcientífica.shtm

Padrón, J. (2006). Investigar, reflexionar y actuar en la práctica docente. Recuperado el 18 de abril de 2008 de http//padron.entretemas.com/ InvAplicada/index.htm

Salkind, N. (1999). Métodos de investigación. México: Prentice Hall.

Samaja, J. (2002). Epistemología y metodología. Buenos Aires: Eudeba.

Universidad de Costa Rica, Facultad de Educación, Escuela de Orientación y Educación Especial. Comisión de Estudios de Posgrado del programa de Maestría en Orientación. (2007). Acta No.6-2007 del 11-06. San José, Costa Rica. Manuscrito sin publicar.

Universidad de Costa Rica, Sistema de Estudios de Posgrado [SEP]. (2007). Lineamientos específicos para los trabajos finales de investigación aplicada de las maestrías profesionales. S. J., Costa Rica. Manuscrito sin publicar.

Vargas, Z. R. (1991). La investigación participativa: Como utilizarla en orientación. Revista Educación, 15(2), 103-110.

Weiss, C. (1987). Investigación evaluativa. México: Trillas. 
